\title{
Helmet noninvasive ventilation for COVID-19 patients (Helmet-COVID): statistical analysis plan for a randomized controlled trial
}

Yaseen Arabi ${ }^{1 *}$ (D), Sara Aldekhyll, Saad Al Qahtani ${ }^{1}$, Hasan M. Al-Dorzi ${ }^{1}$, Sheryl Ann Abdukahil ${ }^{1}$, Jesna Jose ${ }^{3}$, Mohammad Khulaif Al Harbi ${ }^{4}$, Husain Al Haji ${ }^{5}$, Mohammed Al Mutairi ${ }^{5}$, Omar Al Zumai ${ }^{5}$, Eman Al Qasim ${ }^{6}$, Wedyan Al Wehaibi ${ }^{1}$, Mohammed Alshahrani ${ }^{7}$, Talal Albrahim ${ }^{8}$, Ahmed Mady ${ }^{9,10}$, Ali Al Bshabshe ${ }^{11}$, Zohair Al Aseri ${ }^{12}$, Zainab Al Duhailib ${ }^{13}$, Ayman Kharaba ${ }^{14}$, Rakan Alqahtani ${ }^{15}$, Haifa Algethamy ${ }^{16}$, Omar Alfaris ${ }^{17}$, Omar Alnafel ${ }^{18}$, Abdulrahman A. Al-Fares ${ }^{19}$ and Haytham Tlayjeh ${ }^{1}$

\begin{abstract}
Background: Noninvasive respiratory support is frequently needed for patients with acute hypoxemic respiratory failure due to coronavirus disease 19 (COVID-19). Helmet noninvasive ventilation has multiple advantages over other oxygen support modalities but data about effectiveness are limited.

Methods: In this multicenter randomized trial of helmet noninvasive ventilation for COVID-19 patients, 320 adult ICU patients (aged $\geq 14$ years or as per local standards) with suspected or confirmed COVID-19 and acute hypoxemic respiratory failure (ratio of arterial oxygen partial pressure to fraction of inspired oxygen $<200$ despite supplemental oxygen with a partial/non-rebreathing mask at a flow rate of $10 \mathrm{~L} / \mathrm{min}$ or higher) will be randomized to helmet noninvasive ventilation with usual care or usual care alone, which may include mask noninvasive ventilation, high-flow nasal oxygen, or standard oxygen therapy. The primary outcome is death from any cause within 28 days after randomization. The trial has $80 \%$ power to detect a $15 \%$ absolute risk reduction in 28 -day mortality from 40 to $25 \%$. The primary outcome will be compared between the helmet and usual care group in the intention-to-treat using the chi-square test. Results will be reported as relative risk and 95\% confidence interval. The first patient was enrolled on February 8, 2021. As of August 1, 2021, 252 patients have been enrolled from 7 centers in Saudi Arabia and Kuwait.
\end{abstract}

Discussion: We developed a detailed statistical analysis plan to guide the analysis of the Helmet-COVID trial, which is expected to conclude enrollment in November 2021.

Trial registration: ClinicalTrials.gov NCT04477668. Registered on July 20, 2020

Keywords: Noninvasive ventilation, Helmet noninvasive ventilation, COVID-19, Statistical analysis plan

\footnotetext{
* Correspondence: yaseenarabi@yahoo.com

Related article: https://doi.org/10.1136/bmjopen-2021-052169.

'Intensive Care Department, Ministry of National Guard Health Affairs, King

Abdullah International Medical Research Center, King Saud Bin Abdulaziz

University for Health Sciences, Riyadh, Saudi Arabia

Full list of author information is available at the end of the article
}

C The Author(s). 2022 Open Access This article is licensed under a Creative Commons Attribution 4.0 International License, which permits use, sharing, adaptation, distribution and reproduction in any medium or format, as long as you give appropriate credit to the original author(s) and the source, provide a link to the Creative Commons licence, and indicate if changes were made. The images or other third party material in this article are included in the article's Creative Commons licence, unless indicated otherwise in a credit line to the material. If material is not included in the article's Creative Commons licence and your intended use is not permitted by statutory regulation or exceeds the permitted use, you will need to obtain permission directly from the copyright holder. To view a copy of this licence, visit http://creativecommons.org/licenses/by/4.0/. The Creative Commons Public Domain Dedication waiver (http://creativecommons.org/publicdomain/zero/1.0/) applies to the data made available in this article, unless otherwise stated in a credit line to the data. 


\section{Background}

Acute hypoxemic respiratory failure is a common feature of severe coronavirus disease 19 (COVID-19) [1] and frequently requires respiratory support. As invasive mechanical ventilation carries high morbidity and mortality, other respiratory modalities, such as high-flow nasal oxygen (HFNO) and noninvasive ventilation (NIV) delivered via face mask or helmet, have been suggested and increasingly practiced. Helmet NIV has multiple advantages over other modalities that may include more effective seal, less transmission of the virus, more effective delivery of positive endexpiratory pressure (PEEP), and greater tolerance $[2,3]$. Helmet NIV has been investigated as a treatment in adult patients with acute hypoxemic respiratory failure [4-8]. A network meta-analysis of 25 studies that included 3804 patients with acute hypoxemic respiratory failure for reasons other than COVID-19 found significantly lower risks of intubation (risk ratio, 0.26; 95\% credible interval, 0.14-0.46) and mortality (risk ratio, 0.40; 95\% credible interval, $0.24-$ 0.63) with helmet NIV compared with standard oxygen therapy [9]. Recently, a randomized controlled trial (RCT) compared the early application of $48 \mathrm{~h}$ of helmet NIV to high-flow nasal oxygen (HFNO) in 109 patients with moderate to severe hypoxemia (ratio of partial pressure of arterial oxygen to fraction of inspired oxygen $\left(\mathrm{PaO}_{2}: \mathrm{FiO}_{2}\right)$ ratio $\leq 200$ ) and showed no difference in the number of days free of respiratory support at 28 days (primary outcome) with a significantly lower incidence of intubation and a higher number of invasive mechanical ventilation-free days at 28 days in the helmet NIV group [10].

The helmet noninvasive ventilation for COVID-19 patients (Helmet-COVID) trial is a concealed, unblinded multicenter RCT that evaluates the effect of helmet NIV plus usual care compared to usual care alone on 28-day mortality in patients with acute hypoxic respiratory failure due to COVID-19. The full trial protocol has been published previously [11].

In this manuscript, we describe the statistical analysis plan (SAP) of the Helmet-COVID trial. The report describes the procedures for the primary and secondary analyses. All analyses were prospectively defined as the SAP was finalized during trial implementation. The SAP was written by the principal investigator and members of the Steering Committee, who will remain blinded to the study results until all patients have been recruited and the database has been locked. Participant recruitment is expected to be completed in November 2021. The final study report will follow the CONSORT (Consolidated Standards of Reporting Trials) 2010 guidelines for reporting RCTs $[12,13]$.

\section{Methods}

\section{Study design}

The Helmet-COVID trial will enroll 320 critically ill patients in Saudi Arabia and Kuwait. The study has been approved by the Institutional Review Boards of the participating sites. The trial is registered in ClinicalTrials. gov (NCT04477668). The study is sponsored and funded by King Abdullah International Medical Research Center (protocol number RC20/306/R), Riyadh, Saudi Arabia. The sponsor has no role in the study design, management, or analysis.

\section{Study population}

All adult (adult ICU cut-off age) patients admitted to the ICU with suspected or confirmed COVID-19 by reverse transcription-polymerase chain reaction (RT-PCR) will be screened for eligibility. Inclusion criteria include acute hypoxemic respiratory failure $\left(\mathrm{PaO}_{2}: \mathrm{FiO}_{2}\right.$ ratio < 200 despite supplemental oxygen with a partial/non-rebreathing mask at a flow rate of $10 \mathrm{~L} / \mathrm{min}$ or above) with intact airway protective gag reflex and ability to follow instructions. Exclusion criteria include imminent intubation and the requirement for more than one vasopressor. A full list of inclusion and exclusion criteria is described in the published protocol [11]. Enrolled patients will be randomized using a concealed online system to helmet NIV with usual care or usual care alone at a 1:1 ratio. Usual care may include mask NIV, HFNO, or standard oxygen therapy. Randomization will be stratified by site. Helmet NIV will be delivered in pressure support mode, with initial settings of pressure support of $8-10 \mathrm{~cm} \mathrm{H} \mathrm{H}_{2} \mathrm{O}$, PEEP of $10 \mathrm{~cm} \mathrm{H}_{2} \mathrm{O}$ with $\mathrm{FiO}_{2}$ of $100 \%$, targeting flow rate of $\geq 50 \mathrm{~L} / \mathrm{min}$ with an inspiratory rise time of $50 \mathrm{~ms}$, and end flow/ cycling off of $50 \%$ of maximal inspiratory flow. The settings can be adjusted according to the study protocol. The management of respiratory support in the usual care group including mask NIV is at the discretion of the treating team.

A flow diagram will be constructed according to the CONSORT guidelines (Fig. 1). We will report the number of patients who were screened, met inclusion or exclusion criteria, and were eligible but not enrolled with reasons for non-enrollment. We will report the number of patients who were randomized to each group, received the allocated intervention, withdrawn/lost-to-follow-up with reasons, and included in the final analysis.

The intention-to-treat population consists of all randomized patients and will be used for the primary analysis. All randomized patients will be included regardless of whether they receive or do not receive the allocated intervention. All patients randomized with suspected COVID-19 will remain in the study, even if they tested negative for COVID-19 after enrollment. Postenrollment exclusion from the intention-to-treat analysis will be restricted to the withdrawal of consent to use trial data by the patient or surrogate decision-maker 


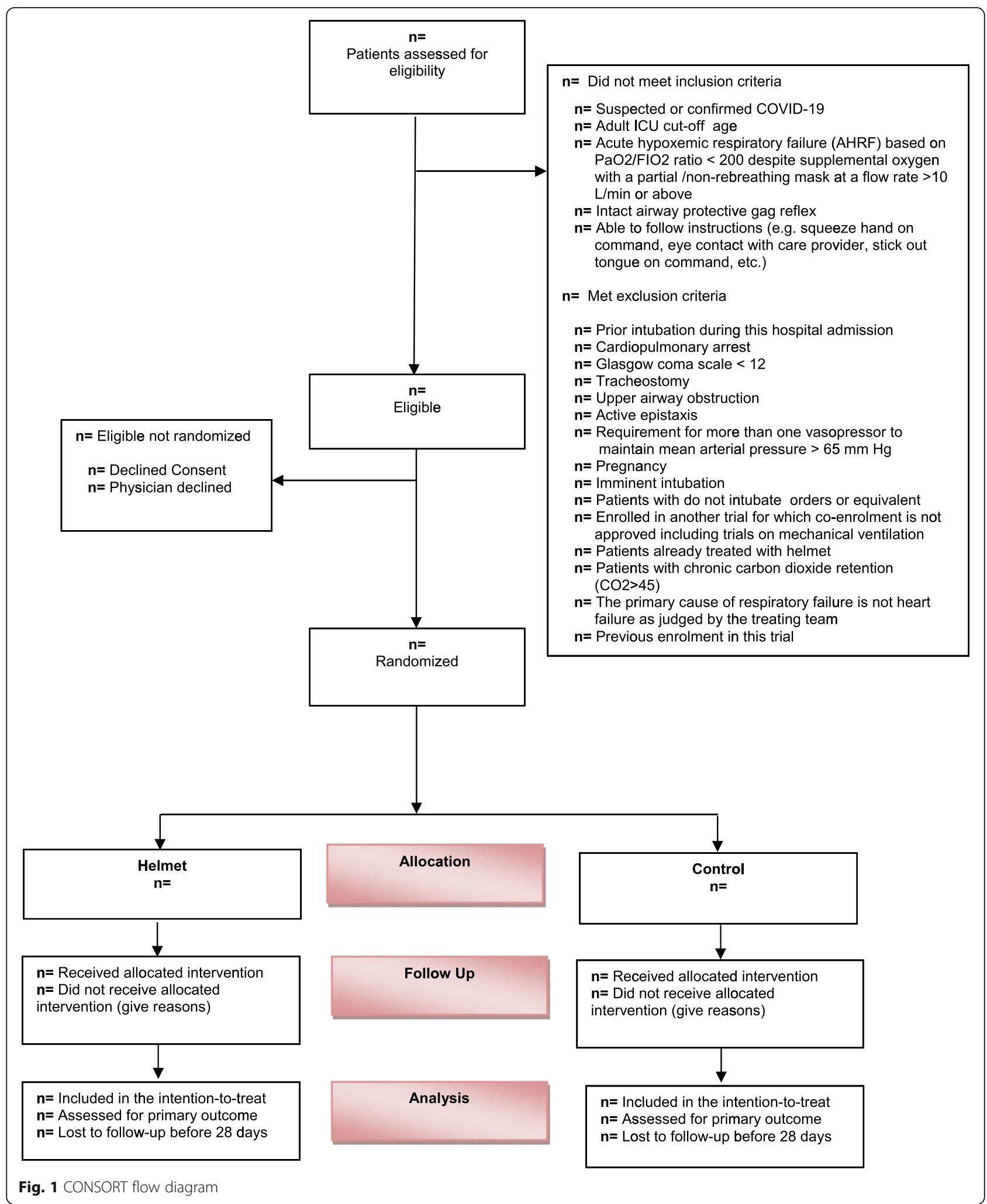


(SDM) or wrong randomization (for example, randomization of an ineligible patient in error). However, if the patient or SDM withdraws consent for trial participation but permits collection and use of data, the patient will be included in the intention-to-treat analysis. We plan to enroll additional patients to compensate for patients who are excluded post-randomization, so the sample size of 320 patients in the intention-to-treat cohort is reached.

The per-protocol population consists of all randomized patients who receive respiratory support as per the allocated group (helmet NIV in the helmet NIV group, and no helmet NIV in the usual care group). Patients will be considered to have received helmet NIV if the device was applied for $1 \mathrm{~h}$ or more.

\section{Data}

\section{Baseline characteristics}

We will present baseline characteristics in patients randomized to the helmet NIV and usual care groups in the intention-to-treat cohort (Supplement Table S1). We will report in the two groups patients' age, sex, height, weight, body mass index, location before ICU admission (emergency room, hospital ward, other hospitals (ICU or ward), others), Acute Physiology and Chronic Health Evaluation (APACHE) II, Sequential Organ Failure Assessment (SOFA) score, comorbidities (any chronic comorbidity, chronic cardiac, pulmonary disease, renal, liver, and neurological diseases, diabetes, any malignancy including leukemia or lymphoma and metastatic solid tumor, AIDS/HIV, rheumatologic diseases, others). Because we are enrolling patients with confirmed or suspected COVID-19, we will report whether the patient is eventually confirmed to have COVID-19. We will report physiologic parameters before randomization $\left(\mathrm{PaO}_{2}: \mathrm{FiO}_{2}\right.$ ratio, partial pressure of carbon dioxide $\left(\mathrm{PaCO}_{2}\right)$, and $\mathrm{pH}$ ) and the number of quadrants with infiltrates on the chest radiograph. We will report respiratory support at baseline (HFNO, mask NIV, standard oxygen therapy). We will document respiratory rate and whether the patient is treated with awake prone positioning. We will document the number of days from the onset of symptoms to the emergency room and ICU admission and the number of days from ICU admission to randomization. We will report non-respiratory organ support, including vasopressor therapy and renal replacement therapy.

\section{Intervention data}

For each $24 \mathrm{~h}$ in the first $96 \mathrm{~h}$, we will report in each group the details regarding helmet NIV (number of hours used, highest pressure support level, and PEEP). Throughout the first 28 days, we will document the number of days with helmet treatment $(>1 \mathrm{~h})$ and the total hours of helmet NIV. Non-tolerance to helmet NIV is defined as the need to remove the helmet because of patient preference or evidence of clinical deterioration. We will document other reasons for discontinuation of the helmet NIV (clinical improvement, the need for intubation, helmet removal due to change in goals of care or death while on helmet). We will document respiratory support after discontinuation of helmet NIV (mask NIV, HFNO, other oxygen devices, intubation). Violations to the study protocol will be documented including the use of NIV helmet in the usual care group and lack of attempt to use helmet NIV in the intervention group (Supplement Table S2).

\section{Co-interventions}

In both groups, we will document the use of other respiratory support modalities during the first 4 days (mask NIV with highest pressure support and PEEP, HFNO with flow rate, other oxygen devices, awake prone position), arterial blood gases, and fluid intake and output (Supplement Table S2).

\section{Physiologic variables during the intervention}

For the 28 days, we will document modalities of respiratory support, the use of sedation while not intubated (dexmedetomidine is permitted in the protocol), renal replacement therapy, and vasopressors/inotrope therapy. We will document the use of COVID-19 treatments, including corticosteroids, IL- 6 receptor antagonists, and antiviral therapy. We will document serial arterial oxygen saturation $\left(\mathrm{SaO}_{2}\right) / \mathrm{FiO}_{2}$ ratio, fluid balance, and serial SOFA scores (Supplement Table S2).

\section{Primary outcome}

The primary outcome is all-cause 28-day mortality. The primary outcome tests the primary hypothesis that helmet NIV reduces 28-day mortality (Supplement Table S3).

\section{Secondary outcomes}

A detailed list of secondary outcomes with definitions has already been published and is outlined in Supplement Table S4. These secondary outcomes can be grouped as follows:

1. Mortality outcomes
a. ICU mortality
b. Hospital mortality

2. Endotracheal intubation. We will document time to intubation, reasons for intubation as determined by the treating team (neurologic deterioration that is not attributed to sedation, persistent or worsening respiratory failure of NIV such as $\mathrm{SaO} 2<88 \%$, respiratory rate $>36 / \mathrm{min}, \mathrm{PaO}_{2}: \mathrm{FiO}_{2}$ ratio $<100$ or persistent requirement of $\mathrm{FiO}_{2} \geq 70 \%$, intolerance 
of mask or helmet NIV, airway bleeding, copious respiratory secretions, respiratory acidosis with $\mathrm{pH}$ $<7.25$, hemodynamic instability, or significant radiological worsening). We will document mechanical ventilation parameters in the first $24 \mathrm{~h}$ after intubation (peak airway pressure, plateau pressure, $\mathrm{PEEP}$ in $\mathrm{cm}_{2} \mathrm{O}$ ), $\mathrm{FiO}_{2}$, tidal volume, and respiratory rate. We will also document oxygen rescue therapies during invasive mechanical ventilation (neuromuscular blocker infusion, recruitment maneuvers, inhaled nitric oxide, prone positioning, extracorporeal membrane oxygenation $(E C M O))$. We will report the percentage of patients who underwent tracheostomy.

3. Continuous outcomes

a. ICU-free days at day 28

b. Hospital length of stay (LOS)

c. Invasive mechanical ventilation-free days at day 28

d. Renal replacement therapy-free days at day 28

e. Vasopressor-free days at day 28

4. Safety outcomes

a. Skin injury at the nose, face, neck, and axillae, with the highest stage during the intervention period. We will use the stages as per the National Pressure Ulcer Advisory Panel [14]: stage I: non-blanchable erythema, stage II: partial thickness, stage III: full-thickness skin loss, and stage IV: full-thickness tissue loss

b. Barotrauma, including pneumothorax, mediastinal air or subcutaneous emphysema

c. Cardiovascular events

d. Device complications (such as helmet deflation or malfunction)

e. Serious adverse events (SAEs)

5. Follow-up study: There will be a follow-up of enrolled patients at day 180 about vital status, functional status (EuroQoL (EQ)-5D-5L), by an unblinded assessor, which is planned to be reported separately. For patients who have been discharged from the hospital before day 180, follow-up will be conducted by telephone.

We will also report protocol violations (Supplement Table S6).

\section{Statistical analysis}

Details of sample size calculation have already been published in the study protocol [11]. The sample size of 320 provides a power of $80 \%$ to detect a $15 \%$ absolute risk reduction in 28-day mortality from 40 to $25 \%$. Categorical variables will be reported as numbers and frequencies and will be compared between the study groups using the chi-square test or Fisher's exact test.
Continuous variables will be reported as means and standard deviations or medians and the first and third quartiles (Q1-Q3) and will be compared between the study groups using the Student's $t$-test or the Wilcoxon-Mann-Whitney test, as judged appropriate by normality testing using Shapiro-Wilk test. For serial measurements (such as $\mathrm{SaO}_{2}: \mathrm{FiO} 2$ ratio, fluid balance, and serial SOFA), we will test the change over time and the difference between the two study groups over time using generalized linear mixed effect models by considering the link function of logit to incorporate the binary nature of the response variable and link function of $\log$ for score outcomes, with no imputation for missing values. To adjust for multiple testing for secondary outcomes and subgroup analyses, we will use the false discovery rate (FDR) as described by Benjamini and Hochberg [15]. We will report associations as relative risk (RR) or hazard ratio (HR) with $95 \%$ confidence intervals $(\mathrm{CI})$ or beta coefficient and $95 \% \mathrm{CI}$ as appropriate. We will compare dyspnea scores and device discomfort scores using generalized linear mixed models, incorporating fixed effects of treatment, time, and the treatment by time interaction and random effects of patient and center. Tests will be two-sided and at the 5\% significance level. All statistical analyses will be conducted using the SAS software version 9.4 (SAS Institute, Cary, NC, USA). The statistical analysis remains blinded to the research team until completion of primary outcome data on the study population and will be performed by the study biostatistician. A summary of the analysis plan is provided in Table 1 .

\section{Analysis of primary outcome}

The primary outcome will be compared in the intention-to-treat using the chi-square test. Results will be reported as RR with 95\% CI. We will use an unadjusted Cox proportional hazard model as a secondary analysis tool, and the results will be reported as hazard ratio (HR) and 95\% CI. We will use Kaplan-Meier survival function estimates to assess proportional hazards for categorical covariates. Moreover, we will be using the supremum test of the null hypothesis that the observed pattern of martingale residuals is not different from the expected pattern [16]. A very small $p$-value $(\leq 0.05)$ suggests a violation of proportional hazards. Kaplan-Meier curves will be generated for the two study groups and a log-rank test will be used to compare distributions. Although imbalances in baseline characteristics are unlikely with the large sample size, we will conduct an adjusted logistic regression model to adjust for the following factors (defined a priori): enrolment center, respiratory support at baseline (mask NIV support versus others), $\mathrm{PaO}_{2}: \mathrm{FiO}_{2}$ ratio, body mass index $>30 \mathrm{~kg} / \mathrm{m}^{2}$, age, APACHE II score, and time (time of enrolling the 
Table 1 Summary of the analysis plan

\begin{tabular}{|c|c|c|}
\hline Variables & Intention-to-treat cohort & Per-protocol cohort \\
\hline $\begin{array}{l}\text { Baseline } \\
\text { characteristics }\end{array}$ & No statistical comparisons & None \\
\hline $\begin{array}{l}\text { Intervention and } \\
\text { co-interventions }\end{array}$ & $\begin{array}{l}\text { Chi-square, Fisher's exact test, Wilcoxon-Mann-Whitney test, } \\
\text { t-test as applicable }\end{array}$ & None \\
\hline Primary outcome & $\begin{array}{l}\text { 1. Primary analysis: chi-square or Fisher's exact test. } \\
\text { Report relative risk. } \\
\text { 2. Secondary analyses: unadjusted Cox proportional } \\
\text { analysis, KM curves, adjusted logistic regression }\end{array}$ & $\begin{array}{l}\text { 1. Primary analysis: chi-square or Fisher's exact test. } \\
\text { Report relative risk. } \\
\text { 2. Secondary analyses: unadjusted Cox proportional analysis, } \\
\text { KM curves, adjusted logistic regression }\end{array}$ \\
\hline $\begin{array}{l}\text { Secondary } \\
\text { outcomes-categorical }\end{array}$ & Chi-square or Fisher's exact test. Report relative risk & Chi-square or Fisher's exact test. Report relative risk \\
\hline $\begin{array}{l}\text { Secondary } \\
\text { outcomes-continuous }\end{array}$ & Generalized linear mixed models. Report beta estimate & Generalized linear mixed models. Report beta estimate \\
\hline $\begin{array}{l}\text { Safety outcomes } \\
\text { and other variables }\end{array}$ & $\begin{array}{l}\text { Chi-square or Fisher's exact test. Report relative risk. } \\
\text { For serial measurements, generalized linear mixed effect models }\end{array}$ & None \\
\hline Subgroup analyses & $\begin{array}{l}\text { Chi-square or Fisher's exact test. Report relative risk. } \\
\text { Tests of interaction }\end{array}$ & None \\
\hline 180-day follow-up & $\begin{array}{l}\text { Chi-square or Fisher's exact test for 180-day mortality. } \\
\text { Report relative risk } \\
\text { Generalized linear mixed models for EQ-5D-5L ad VAS. } \\
\text { Report beta estimate }\end{array}$ & $\begin{array}{l}\text { Chi-square or Fisher's exact test for 180-day mortality. } \\
\text { Report relative risk } \\
\text { Generalized linear mixed models for EQ-5D-5L ad VAS. } \\
\text { Report beta estimate }\end{array}$ \\
\hline
\end{tabular}

first half of the cohort versus the second half); the latter will be included to account for the changes in outcomes of COVID-19 patients during the pandemic.

\section{Analysis of secondary outcomes}

Secondary outcomes will be compared in the intentionto-treat cohort only. Categorical outcomes will be compared in the intention-to-treat using a chi-square test. Results will be reported as RR with 95\% CI. Continuous outcomes will be compared using generalized linear mixed models. Results will be reported as beta estimates with $95 \%$ CI.

\section{Subgroup analyses}

The primary outcome will be compared in the intention-to-treat cohort only, in the following a priori defined subgroups using a chi-square test.

1. $\mathrm{PaO}_{2}: \mathrm{FiO}_{2}$ ratio $101-200$ and $\mathrm{PaO}_{2}: \mathrm{FiO}_{2}$ ratio $<100$

2. Obese patients (body mass index $>30 \mathrm{~kg} / \mathrm{m}^{2}$ ) and patients with a body mass index of $\leq 30 \mathrm{~kg} / \mathrm{m}^{2}$

3. Patients aged $>65$ years and $\leq 65$ years

4. APACHE II score higher or lower than the median of enrolled patients

5. Patients who were at the time of enrollment on mask NIV versus other types of respiratory support

Results will be reported using RR and 95\% CI. We will report the results of the test of interactions for these subgroups (Supplement Table S5).

\section{Sensitivity analyses}

We will compare the primary outcome between the helmet NIV and usual care groups in the per-protocol cohorts (effectiveness analysis). If patients with suspected COVID-19 who tested negative constituted more than $5 \%$ of the study population, we will carry a sensitivity analysis excluding these patients.

\section{0-day follow-up study}

In a follow-up report, we will compare 180-day mortality and the EuroQol (EQ-5D-5L) [17] at 6-month scores after randomization between the two study groups. The EQ-5D-5L scores will be converted into a single index value that generates a measure of utility ranging from -0.111 to 1.000 (1.000 indicates full health) using an online tool $[18,19]$. We will use generalized linear mixed models to compare EQ-5D-5L index values and VAS scores between the two groups by incorporating treatment, baseline values, and random patient and center effects in the model.

\section{Interim analyses}

The interim test statistics will be conducted for the primary outcome. Two formal interim analyses are planned when $33 \%$ and $67 \%$ of the sample size are reached. The trial may be stopped for safety $(p<$ $0.01)$ or effectiveness $(p<0.001)$ both evaluating the primary outcome (28-day mortality) but there will be no plans to terminate the trial for futility. We will account for alpha spending by the O'Brien-Fleming method and the final $p$-value will be considered at 0.048 [20]. 


\section{Discussion}

Several studies have investigated helmet NIV as a treatment for acute hypoxic respiratory failure [4-8]. A systematic review of RCTs and observational studies found that helmet NIV was associated with lower hospital mortality (odds ratio, 0.43; 95\% CI, 0.26-0.69), intubation rate (odds ratio, $0.32 ; 95 \%$ CI, $0.21-0.47$ ), and complications (odds ratio, 0.6; 95\% CI, 0.4-0.92) compared with controls [21]. A meta-analysis of four RCTs (377 patients) showed that helmet NIV significantly increased the $\mathrm{PaO}_{2}: \mathrm{FiO}_{2}(+73.4 ; 95 \% \mathrm{CI}, 43.9-102.9)$ and decreased the arterial $\mathrm{CO}_{2}$ levels, intubation rate $(\mathrm{RR}, 0.21$; 95\% CI, 0.11-0.40), and in-hospital mortality rate (RR, 0.22; 95\% CI, 0.09-0.50) compared to standard oxygen therapy [22]. A network meta-analysis of 25 studies that included 3804 patients with acute hypoxemic respiratory failure for reasons other than COVID-19 found significantly lower intubation risks with helmet NIV compared with standard oxygen therapy [9]. The advantages of helmet NIV over other oxygen support modalities are thought to be more prominent in patients with COVID19. This led to the design and conduct of multiple RCTs $[10,23]$. Recently, one trial $(n=110)$ showed no difference in the number of days free of respiratory support at 28 days (primary outcome) between helmet NIV and HFNO [10].

The trial is unblinded given the nature of the intervention. The use of an objective, all-cause mortality as the primary outcome mitigates concerns regarding outcome assessment. The lack of blinding for the long-term quality of life outcomes is a limitation.

As the efficacy of helmet NIV to improve outcomes in severe acute hypoxemic respiratory failure due to COVID19 pneumonia has not been established, the aim of the Helmet-COVID trial is to compare the effectiveness of helmet NIV compared to usual care on 28-day mortality in patients with acute hypoxic respiratory failure from COVID-19. The results of the trial and the possibility of contributing to individual patient meta-analysis would likely help address this important question.

\section{Trial status}

The first patient was enrolled on February 8, 2021, and enrollment is expected to be concluded in November 2021.

\section{Conclusion}

The Helmet-COVID trial evaluates whether helmet NIV improves the outcomes of critically ill patients with acute hypoxemic respiratory failure due to COVID-19. It is expected to provide evidence that will inform practice regarding the use of helmet NIV for respiratory support in these patients and contribute to future clinical practice guidelines.

\section{Abbreviations}

$\mathrm{Cl}$ : Confidence interval; HR: Hazard ratio; ICU: Intensive care unit;

IQR: Interquartile range; LOS: Length of stay; RT-PCR: Reverse transcriptionpolymerase chain reaction; RCT: Randomized controlled trial; RR: Relative risk; RRR: Relative risk reduction; SAP: Statistical analysis plan

\section{Supplementary Information}

The online version contains supplementary material available at https://doi. org/10.1186/s13063-021-05988-x.

Additional file 1. Supplementary tables and figures

\section{Acknowledgements}

The authors would like to thank all the participating patients and their families, as well as the members of the Data Safety \& Monitoring Board: Chair: Dr. Nicholas S. Hill (Professor of Medicine, Chief of Pulmonary, Critical Care and Sleep Division, Tufts Medical Center, Boston, Massachusetts, USA), DSM members: Dr. Stefano Nava (Professor of Respiratory Medicine

University of Bologna, Chief of the Respiratory and Critical Care, Alma Mater Studiorum University of Bologna. IRCCS Sant'Orsola Hospital, Respiratory and Crtical Care Unit University of Bologna, Italy), James Mojica, MD (Vice Chief \& Clinical Director of the Division of Pulmonary \& Critical Care Medicine; Massachusetts General Hospital, USA) and Dr. Michael Harhay (Assistant Professor of Epidemiology and Medicine-Pulmonary and Critical Care, Department of Biostatistics, Epidemiology and Informatics, University of Pennsylvania, USA).

\section{Authors' contributions}

YA is the principal investigator and participated in the project concept, design, final approval, and manuscript preparation, review, and submission. SD, SQ, HD, SA, JJ, MH, HH, MM, OZ, EQ, WW, MS, TI, AM, AB, ZA, ZD, AK, RQ, $\mathrm{HG}, \mathrm{OF}, \mathrm{ON}, \mathrm{AF}$, and $\mathrm{HT}$ participated in the critical revision, final approval of the protocol, and manuscript preparation, review, and submission. All authors agree to be accountable for the accuracy and integrity of the work. The authors read and approved the final manuscript.

\section{Funding}

The study is funded and sponsored by King Abdullah International Medical Research Center (RC 20/306/R), Riyadh, Saudi Arabia. The study sponsor does not have any role in the study design, collection, management, analysis, and interpretation of data as well as the writing of the report.

\section{Availability of data and materials}

The dataset will be available from the corresponding author upon reasonable request in accordance with the regulations of King Abdullah International Medical Research Center, Riyadh, Saudi Arabia.

\section{Declarations}

Ethics approval and consent to participate

The study has been approved by the Institutional Review Board (IRB) of the Ministry of National Guard Health Affairs (MNGHA) and the respective Institutional Review Boards of all the other centers.

\section{Consent for publication}

Not applicable

Competing interests

The authors declare that they have no competing interests.

\section{Author details}

${ }^{1}$ Intensive Care Department, Ministry of National Guard Health Affairs, King Abdullah International Medical Research Center, King Saud Bin Abdulaziz University for Health Sciences, Riyadh, Saudi Arabia. ${ }^{2}$ College of Medicine, King Saud Bin Abdulaziz University for Health Sciences, King Abdullah International Medical Research Center, Ministry of National Guard Health Affairs, Riyadh, Saudi Arabia. ${ }^{3}$ Bioinformatics and Biostatistics Department, King Abdullah International Medical Research Center, King Saud Bin Abdulaziz University for Health Sciences, Ministry of National Guard Health 
Affairs, Riyadh, Saudi Arabia. ${ }^{4}$ Department of Anesthesia, Ministry of Nationa Guard Health Affairs, King Abdullah International Medical Research Center, King Saud Bin Abdulaziz University for Health Sciences, Riyadh, Saudi Arabia. ${ }^{5}$ Respiratory Services Department, Ministry of National Guard Health Affairs, King Abdullah International Medical Research Center, King Saud Bin Abdulaziz University for Health Sciences, Riyadh, Saudi Arabia. ${ }^{6}$ Research Office, King Abdullah International Medical Research Center, King Saud Bin Abdulaziz University for Health Sciences, Ministry of National Guard Health Affairs, Riyadh, Saudi Arabia. 'Department of Emergency and Critical Care, King Fahad Hospital of the University, Imam Abdulrahman Bin Faisal University, Al Khobar, Kingdom of Saudi Arabia. ${ }^{8}$ Department of Critical Care, King Fahad Hospital of the University, Imam Abdulrahman Bin Faisal University, Al Khobar, Kingdom of Saudi Arabia. ${ }^{9}$ Intensive Care Department, King Saud Medical City, Riyadh, Saudi Arabia. ${ }^{10}$ College of Medicine, Tanta University, Tanta, Egypt. ${ }^{11}$ Department of Critical Care Medicine, King Khalid University, Aseer Central Hospital, Abha, Kingdom of Saudi Arabia.

${ }^{12}$ Emergency and Intensive Care Departments, College of Medicine, King Saud University, Riyadh, Saudi Arabia. ${ }^{13}$ Adult Critical Care Department, King Faisal Specialist Hospital \& Research Center, Riyadh, Saudi Arabia.

${ }^{14}$ Pulmonary \& Critical Care Departments, King Fahad Hospital Madinah Critical Care Units, Madinah, Saudi Arabia. ${ }^{15}$ Department of Critical Care, College of Medicine, King Saud University, Riyadh, Saudi Arabia.

${ }^{16}$ Department of Anesthesia and Critical Care, King Abdulaziz University, King Abdulaziz University Hospital, Jeddah, Saudi Arabia. ${ }^{17}$ Respiratory Services Department, Ministry of National Guard Health Affairs, King Abdullah International Medical Research Center, King Saud Bin Abdulaziz University for Health Sciences, Riyadh, Saudi Arabia. ${ }^{18}$ Internal Medicine and Intensive Care Department, King Salman Specialist Hospital, Hail, Saudi Arabia.

${ }^{19}$ Department of Anesthesia, Critical Care Medicine and Pain Medicine, Al-Amiri Hospital, Ministry of Health, Kuwait, Kuwait.

Received: 2 September 2021 Accepted: 27 December 2021 Published online: 02 February 2022

\section{References}

1. Richardson S, Hirsch JS, Narasimhan M, Crawford JM, McGinn T, Davidson $\mathrm{KW}$, et al. Presenting characteristics, comorbidities, and outcomes among 5700 patients hospitalized with COVID-19 in the New York City area. Jama. 2020;323(20):2052-9. https://doi.org/10.1001/jama.2020.6775.

2. Cabrini L, Landoni G, Zangrillo A. Minimise nosocomial spread of 2019-nCoV when treating acute respiratory failure. Lancet. 2020;395(10225):685. https:// doi.org/10.1016/S0140-6736(20)30359-7.

3. Amirfarzan H, Cereda M, Gaulton T, Leissner K, Cortegiani A, Schumann R, et al. Use of helmet CPAP in COVID-19: a practical review. Pulmonology. 2021;:S2531-0437(21):00040-4

4. Brambilla AM, Aliberti S, Prina E, Nicoli F, Del Forno M, Nava S, et al. Helmet CPAP vs. oxygen therapy in severe hypoxemic respiratory failure due to pneumonia. Intensive Care Med. 2014;40(7):942-9. https://doi.org/10.1007/ s00134-014-3325-5.

5. Cosentini R, Brambilla AM, Aliberti S, Bignamini A, Nava S, Maffei A, et al. Helmet continuous positive airway pressure vs oxygen therapy to improve oxygenation in community-acquired pneumonia: a randomized, controlled trial. Chest. 2010;138(1):114-20. https://doi.org/10.1378/chest.09-2290.

6. Patel BK, Wolfe KS, Pohlman AS, Hall JB, Kress JP. Effect of noninvasive ventilation delivered by helmet vs face mask on the rate of endotracheal intubation in patients with acute respiratory distress syndrome: a randomized clinical trial. JAMA. 2016;315(22):2435-41. https://doi.org/10.1 001/jama.2016.6338.

7. Foti G, Sangalli F, Berra L, Sironi S, Cazzaniga M, Rossi G, et al. Is helmet CPAP first line pre-hospital treatment of presumed severe acute pulmonary edema? Intensive care medicine. 2009;35(4):656-62. https://doi.org/10.1007/ s00134-008-1354-7.

8. Squadrone V, Coha M, Cerutti E, Schellino M, Biolino P, Occella P, et al. Continuous positive airway pressure for treatment of postoperative hypoxemia: a randomized controlled trial. Jama. 2005;293(5):589. https://doi. org/10.1001/jama.293.5.589.

9. Ferreyro BL, Angriman F, Munshi L, Del Sorbo L, Ferguson ND, Rochwerg B, et al. Association of noninvasive oxygenation strategies with all-cause mortality in adults with acute hypoxemic respiratory failure: a systematic review and meta-analysis. Jama. 2020;324(1):57-67. https://doi.org/10.1001/ jama.2020.9524.
10. Grieco DL, Menga LS, Cesarano M, Rosà T, Spadaro S, Bitondo MM, et al. Effect of helmet noninvasive ventilation vs high-flow nasal oxygen on days free of respiratory support in patients with COVID-19 and moderate to severe hypoxemic respiratory failure: the HENIVOT randomized clinical trial. JAMA. 2021;325(17):1731-43. https://doi.org/10.1001/jama.2021.4682.

11. Arabi YM, Tlayjeh H, Aldekhyl S, Al-Dorzi HM, Abdukahil SA, Al Harbi MK, et al. Helmet non-invasive ventilation for COVID-19 patients (Helmet-COVID): study protocol for a multicentre randomised controlled trial. BMJ Open. 2021;11(8):e052169. https://doi.org/10.1136/bmjopen-2021-052169.

12. Moher D, Hopewell S, Schulz KF, Montori V, Gotzsche PC, Devereaux PJ, et al. CONSORT 2010 explanation and elaboration: updated guidelines for reporting parallel group randomised trials. BMJ. 2010;340(mar23 1):C869. https://doi.org/10.1136/bmj.c869.

13. Schulz KF, Altman DG, Moher D, Group C. CONSORT 2010 statement: updated guidelines for reporting parallel group randomised trials. BMJ. 2010;340(mar23 1):c332. https://doi.org/10.1136/bmj.c332.

14. Edsberg LE, Black JM, Goldberg M, McNichol L, Moore L, Sieggreen M. Revised national pressure ulcer advisory panel pressure injury staging system: revised pressure injury staging system. Journal of Wound, Ostomy, and Continence Nursing. 2016;43(6):585.

15. Benjamini $Y$, Hochberg $Y$. Controlling the false discovery rate: a practical and powerful approach to multiple testing. J Royal Stat Soc Ser B (Methodol). 1995;57(1):289-300.

16. Lin DY, Wei $\amalg$, Ying Z. Checking the Cox model with cumulative sums of martingale-based residuals. Biometrika. 1993;80(3):557-72. https://doi.org/1 0.1093/biomet/80.3.557.

17. Herdman M, Gudex C, Lloyd A, Janssen M, Kind P, Parkin D, et al. Development and preliminary testing of the new five-level version of EQ-5D (EQ-5D-5L). Qual Life Res. 2011;20(10):1727-36. https://doi.org/10.1007/s1113 6-011-9903-X.

18. EQ-5D-5L Crosswalk Index Value Calculator. Available at: https://euroqol.org/ eq-5dinstruments/eq-5d-5l-about/valuation/crosswalk-index-value-calcula tor/; 2021.

19. Muruganandan S, Azzopardi M, Fitzgerald DB, Shrestha R, Kwan BCH, Lam $\mathrm{DCL}$, et al. Aggressive versus symptom-guided drainage of malignant pleural effusion via indwelling pleural catheters (AMPLE-2): an open-label randomised trial. Lancet Respir Med. 2018;6(9):671-80. https://doi.org/10.101 6/S2213-2600(18)30288-1.

20. O'Brien PC, Fleming TR. A multiple testing procedure for clinical trials. Biometrics. 1979;35(3):549-56. https://doi.org/10.2307/2530245.

21. Liu Q, Gao Y, Chen R, Cheng Z. Noninvasive ventilation with helmet versus control strategy in patients with acute respiratory failure: a systematic review and meta-analysis of controlled studies. Critical Care. 2016;20(1).

22. Luo Y, Luo Y, Li Y, Zhou L, Zhu Z, Chen Y, et al. Helmet CPAP versus oxygen therapy in hypoxemic acute respiratory failure: a meta-analysis of randomized controlled trials. Yonsei Med J. 2016:57(4):936-41.

23. Tverring J, Åkesson A, Nielsen N. Helmet continuous positive airway pressure versus high-flow nasal cannula in COVID-19: a pragmatic randomised clinical trial (COVID HELMET). Trials. 2020;21(1):1-10. https://doi. org/10.1186/s13063-020-04863-5.

\section{Publisher's Note}

Springer Nature remains neutral with regard to jurisdictional claims in published maps and institutional affiliations.

Ready to submit your research? Choose BMC and benefit from:

- fast, convenient online submission

- thorough peer review by experienced researchers in your field

- rapid publication on acceptance

- support for research data, including large and complex data types

- gold Open Access which fosters wider collaboration and increased citations

- maximum visibility for your research: over $100 \mathrm{M}$ website views per year

At BMC, research is always in progress.

Learn more biomedcentral.com/submission 